\title{
Comparison of computed tomography (CT), magnetic resonance imaging (MRI) and contrast-enhanced ultrasound (CEUS) in the evaluation of unclear renal lesions
}

\section{Vergleich von Computertomografie (CT), Magnetresonanztomografie (MRT) und kontrastverstärktem Ultraschall (CEUS) bei der Beurteilung unklarer Nierenläsionen}

\author{
Authors \\ Constantin Arndt Marschner, Johannes Ruebenthaler, Vincent Schwarze, Giovanna Negrão de Figueiredo, Lan Zhang, \\ Dirk Andre Clevert
}

\author{
Affiliation \\ Department of Radiology, Ludwig-Maximilians-University \\ Munich, Germany \\ Key words \\ incidental renal lesion, magnetic resonance tomography, \\ sensitivity, specificity, contrast-enhanced ultrasound, \\ computed tomography
}

received 21.09 .2019

accepted 19.02.2020

Bibliography

Fortschr Röntgenstr 2020; 192: 1053-1058

Published online: 15.4 .2020

DOI $10.1055 / a-1127-3371$

ISSN 1438-9029

(C) 2020. Thieme. All rights reserved.

Georg Thieme Verlag KG, Rüdigerstraße 14,

70469 Stuttgart, Germany

Correspondence

Constantin Arndt Marschner

Klinik und Poliklinik für Radiologie, Ludwig-Maximilians-

University Munich, Marchioninistraße 15, 81377 München, Germany

Tel.: ++ 49/89/4 40073620

Constantin.Marschner@med.uni-muenchen.de

\section{ABSTRACT}

Purpose To compare the sensitivity and specificity of contrast-enhanced ultrasound (CEUS), computed tomography (CT) and magnetic resonance imaging (MRI) in the evaluation of unclear renal lesions to the histopathological outcome.

Materials and methods A total of 255 patients with a single unclear renal mass with initial imaging studies between 2005 and 2015 were included. Patient ages ranged from 18 to 86 with (mean age 62 years; SD \pm 13 ). CEUS ( 255 patients), CT (88 out of 255 patients; $34.5 \%$ ) and MRI (36 out of 255 patients; $14.1 \%$ ) were used for determining malignancy or benignancy and initial findings were correlated with the histopathological outcome.

Results CEUS showed a sensitivity of $99.1 \%$ (95\% confidence interval $(\mathrm{Cl}): 96.7 \%, 99.9 \%)$, a specificity of $80.5 \%(95 \% \mathrm{Cl}$ : $65.1 \%, 91.2 \%$ ), a positive predictive value (PPV) of $96.4 \%$ (95\% Cl: $93.0 \%, 98.4 \%$ ) and a negative predictive value (NPV) of $94.3 \%$ (95\% Cl: $80.8 \%, 99.3 \%$ ). CT showed a sensitivity of $97.1 \%$ (95\% Cl: $89.9 \%, 99.6 \%$ ), a specificity of $47.4 \%$ (95\% Cl: $24.4 \%, 71.1 \%$ ), a PPV of $87.0 \%$ (95\% Cl: $77.4 \%$, $93.6 \%)$ and a NPV of $81.8 \%$ (95\% Cl: $48.2 \%, 97.7 \%)$. MRI showed a sensitivity of $96.4 \%$ (95\% Cl: $81.7 \%$, $99.9 \%$ ), a specificity of $75.0 \%$ (95\% Cl: 34.9\%, 96.8\%), a PPV of $93.1 \%$ (95\% $\mathrm{Cl}: 77.2 \%, 99.2 \%)$ and a NPV of $85.7 \%(95 \% \mathrm{Cl}: 42.1 \%$, $99.6 \%$ ). Out of the 212 malignant lesions a total of 130 clear cell renal carcinomas, 59 papillary renal cell carcinomas, 7 chromophobe renal cell carcinomas, 4 combined clear cell and papillary renal cell carcinomas and 12 other malignant lesions, e. g. metastases, were diagnosed. Out of the 43 benign lesions a total 10 angiomyolipomas, 3 oncocytomas, 8 benign renal cysts and 22 other benign lesions, e. $g$. renal adenomas were diagnosed. Using CEUS, 10 lesions were falsely identified as malignant or benign, whereas 8 lesions were false positive and 2 lesions false negative.

Conclusion CEUS is an useful method which can be additionally used to clinically differentiate between malignant and benign renal lesions. CEUS shows a comparable sensitivity, specificity, PPV and NPV to CT and MRI. In daily clinical routine, patients with contraindications for other imaging modalities can particularly benefit using this method.

Key Points:

- Wide availability

- Safe applicability in patients with known renal insufficiency or allergies to iodine or gadolinium

- Comparable sensitivity, specificity, PPV and NPV to CT and MRT

- May lead to a reduction in interventional radiological or surgical interventions 


\section{Citation Format}

- Marschner CA, Ruebenthaler J, Schwarze V et al. Comparison of computed tomography (CT), magnetic resonance imaging (MRI) and contrast-enhanced ultrasound (CEUS) in the evaluation of unclear renal lesions. Fortschr Röntgenstr 2020; 192: 1053-1058

\section{ZUSAMMENFASSUNG}

Ziel Vergleich der Sensitivität und Spezifität von kontrastverstärktem Ultraschall (CEUS), Computertomografie (CT) und Magnetresonanztomografie (MRT) bei der Beurteilung von unklaren Nierenläsionen mit der histopathologischen Korrelation.

Material und Methoden Zwischen 2005 und 2015 wurden 255 Patienten mit einer einzelnen unklaren Nierenläsion in die vorliegende retrospektive monozentrische Studie eingeschlossen. Das Alter der Patienten lag zwischen 18 und 86 Jahren (Durchschnittsalter 62 Jahre; SD \pm 13 ). CEUS (255 Patienten), CT (88 von 255 Patienten; 34,5\%) und MRT (36 von 255 Patienten; 14,1\%) wurden zur Diagnostik angewendet und die Ergebnisse mit dem histopathologischen Ergebnis korreliert.

Ergebnisse CEUS zeigte eine Sensitivität von 99,1\% (95\%Konfidenzintervall (KI): 96,7\%-99,9\%), eine Spezifität von 80,5\% (95\%-KI: 65,1\%-91,2\%), einen positiven Vorhersagewert (PPV) von 96,4\% (95\%-KI: 93,0\%-98,4\%) und einen negativen Vorhersagewert (NPV) von $94,3 \%$ (95\%-KI:
80,8\%-99,3\%). Die CT zeigte eine Sensitivität von 97,1\% (95\%-KCl: 89,9\%-99,6\%), eine Spezifität von 47,4\% (95\%-Kl: 24,4\%-71,1\%), einen PPV von 87,0\% (95\%-KI: 77,4\%-93,6\%) und einen NPV von 81,8\% (95\%-KI: 48,2\%-97,7\%). Die MRT zeigte eine Sensitivität von 96,4\% (95\%-KI: 81,7\%-99,9\%), eine Spezifität von 75,0\% (95\%-KI: 34,9\%-96,8\%), einen PPV von 93,1\% (95\%-KI: 77,2\%-99,2\%) und einen NPV von 85,7\% (95\%-KI: 42,1\%-99,6\%). Aus den 212 als maligne eingestuften Läsionen wurden insgesamt 130 klarzellige, 59 papilläre, 7 chromophobe und 4 kombinierte klarzellige und papilläre Nierenzellkarzinome sowie 12 weitere maligne Läsionen, z. B. Metastasen, diagnostiziert. Von den 43 benignen Läsionen wurden insgesamt 10 Angiomyolipome, 3 Onkozytome, 8 gutartige Nierenzysten und 22 weitere gutartige Läsionen, z. B. Nierenadenome, diagnostiziert. Mit der CEUS wurden 10 Läsionen fälschlicherweise als maligne oder benigne identifiziert, während 8 Läsionen falsch positiv und 2 Läsionen falsch negativ waren.

Schlussfolgerung CEUS stellt eine hilfreiche Methode dar, die zusätzlich zur Differenzierung zwischen benignen und malignen Nierenläsionen eingesetzt werden kann. CEUS zeigt eine vergleichbare Sensitivität, Spezifität, PPV und NPV gegenüber CT und MRT. In der täglichen klinischen Routine können Patienten mit Kontraindikationen für andere bildgebende Verfahren durch die Anwendung des CEUS besonders profitieren.

\section{Introduction}

Due to the widespread use of ultrasound in all clinical disciplines, there is a high occurrence of unclear, incidentally-discovered renal lesions that raise questions about their characterization and possible preoperative clinical treatment and therefore play an important role in patient management [1-7]. Incidentally-discovered renal lesions are generally benign cysts; thus malignancies can be ruled out with very high probability using the various available imaging techniques $[8,9]$. The most important differential diagnosis for solid or cystic kidney lesions is renal cell carcinoma, which has an incidence rate of $3 \%$ of all malignant neoplasms and is one of the most common tumors of the urinary tract [3]. In the current S3 guidelines for renal cell carcinoma from 2017, contrast-enhanced computed tomography (CE-CT) and contrast-enhanced magnetic resonance imaging (CE-MRI) are the imaging techniques of choice for the characterization and diagnosis of renal cell carcinoma. Contrast-enhanced ultrasound (CEUS) can be used as a supplement for patients with chronic renal insufficiency or a known allergy to contrast media containing iodine or gadolinium [10]. Unlike CE-CT and CE-MRI, ultrasound contrast agents are purely intravascular and do not diffuse into the interstitial space [11-13]. Furthermore, they can be used independently of thyroid and kidney function and show only a low incidence of side effects, the most severe of which is certainly an anaphylactic reaction, which, however, is described in the literature only in 1 in 10,000 cases and can therefore be regarded as uncommon $[14,15]$. Currently CEUS is used in everyday clinical practice as a fast, low-risk and cost-effective method for local diagnosis and staging of renal cell carcinoma [16-19].

Benign and malignant kidney lesions can be distinguished in CEUS since they exhibit a different vascularization pattern to the surrounding healthy renal parenchyma. Unfortunately, some benign kidney lesions such as oncocytomas are hardly distinguishable from malignant lesions such as renal cell carcinomas due to similar enhancement patterns in CEUS, CE-CT and MRI [20, 21]. This retrospective analysis study was performed to compare the sensitivity and specificity of CEUS, CT and MRI in the evaluation of ambiguous renal lesions with the histopathological result as gold standard.

\section{Materials and Methods}

Between 2005 and 2015, a total of 981 patients with a cystic or solid renal lesion that was unclear in preliminary imaging were referred for supplementary CEUS imaging. Within this overall group, a subcohort of 255 patients was retrospectively identified that received histological clarification via FNA/biopsy or surgical resection in subsequent clinical treatment. The diagnosis was made in 10 patients by FNA/biopsy, by surgical resection in the remaining patients. 88 patients (34.5\%) had additional in-domo CT imaging, 36 patients (14.1\%) had additional MRI imaging. Parts of this study population have already been used in previous publications of this research group [18, 22-24].

The imaging performed beforehand consisted of contrast-enhanced CE-CT scans, non-contrast-enhanced CT scans, contrast- 
- Table 1 Characteristic contrast medium behavior in CEUS imaging of solid renal lesions compared to the renal cortex [25].

\begin{tabular}{|l|l|l|l|}
\hline & $\begin{array}{l}\text { arterial contrast medium } \\
\text { behavior }\end{array}$ & $\begin{array}{l}\text { venous contrast medium } \\
\text { behavior }\end{array}$ & late phase \\
\hline clear cell renal cell carcinoma & hyperenhancement & wash-out & persistent wash-out \\
\hline papillary renal cell carcinoma & hypoenhancement & hypoenhancement & hypoenhancement \\
\hline chromophobe renal cell carcinoma & hypoenhancement & hypoenhancement & hypoenhancement \\
\hline oncocytoma & indifferent & indifferent & indifferent \\
\hline angiomyolipoma & indifferent & indifferent & indifferent \\
\hline metastasis/lymphoma & hypoenhancement & hypoenhancement & hypoenhancement \\
\hline pseudotumor & isoenhancement & isoenhancement & isoenhancement \\
\hline pyelonephritis & hypoenhancement & hypoenhancement & hypoenhancement \\
\hline renal abscess & non-enhancing & non-enhancing & non-enhancing \\
\hline
\end{tabular}

enhanced CE-MRI scans or non-contrast-enhanced MRI scans based on the protocols and CT scanners available at the time of examination. In the present patient cohort, the findings were exclusively incidental findings in the primarily performed imaging.

All study data were collected in accordance with the principles of the Helsinki/Edinburgh Declaration of 2002. Oral and written consent of all patients was obtained prior to each CEUS, CT and MRI examination after detailed explanation of the procedure as well as possible risks and complications.

The CEUS examinations were performed on high-end ultrasound systems with current CEUS-specific examination protocols available at the time of the examination (Sequoia/S2000/S3000, Siemens Ultrasound; HDI 5000/iU22/EPIQ 7/Affiniti, Philips Ultrasound; LOGIQ E9, GE Healthcare). The ultrasonic probes used included C6-1 HD, C5-1, C4-1 and V4-1 units. All CEUS examinations were performed and interpreted by a single radiologist with more than 15 years of experience in CEUS. A low mechanical index ( $\mathrm{Ml}$ always $<0.4$ ) was used for the study to avoid accidental destruction of the microbubbles.

A second-generation contrast agent (SonoVue ${ }^{\circledR}$, Bracco, Milan, Italy) was used in all examinations and administered as a bolus injection via a peripheral 20-22 gauge needle. Following the contrast medium injection, the indwelling venous cannula was rinsed with $5-10 \mathrm{ml} 0.9 \%$ saline solution $(0.9 \% \mathrm{NaCl})$.

In the majority of cases 1.6 to $2.4 \mathrm{ml}$ of contrast medium with a range between $1.0 \mathrm{ml}$ and $4.8 \mathrm{ml}$ were administered to this patient population. After injection of the contrast medium, cineloops were created and stored in the in-house image archiving system. The average examination time was 3-5 minutes, and in most cases a single dose of contrast agent was administered. When additive imaging was required, a total of up to three injections of contrast medium was administered. The evaluation of whether the lesion was classified as malignant or benign in the CEUS was based on established qualitative image parameters [25] ( Table 1).

All results of the CEUS, CT and MRI examinations were made without knowledge of the histopathological results at the time of

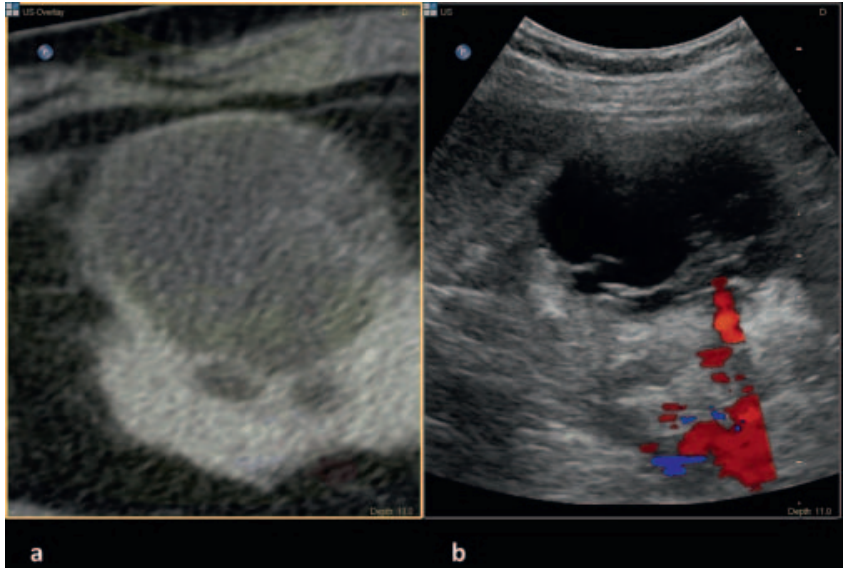

- Fig. 1 Cystic lesion with detection of discrete contrast agent absorption septa in a venous phase contrast enhanced $\mathrm{CT}$ a. In the $\mathbf{B}$-mode imaging and in the color-coded duplex sonography $\mathbf{b}$, an increased vascularization of the septa or the cyst wall cannot be clearly demarcate.

examination and were classified as either benign or malignant (see $>$ Fig. 1-4).

The age of the patients was between 18 and 86 years (mean value: 62 years; standard deviation (SD) \pm 13 ). Of the 255 renal lesions, 212 were classified as malignant (83.1\%) and 43 as benign (16.9\%) in the final histopathological findings. Histological evaluation was performed on material after surgical removal, fine needle biopsy or aspiration.

For statistical analysis, the diagnostic accuracy of CEUS, MRI and $\mathrm{CT}$ was tested with sensitivity, specificity, positive predictive value (PPV) and negative predictive value (NPV). In addition, the $95 \%$ confidence interval $(\mathrm{Cl})$ was calculated for all values. If the first CT or MRI findings were inconclusive and therefore no clear statement about malignancy could be made, the findings for CT or MRI were adjusted to the findings of the CEUS examination. 


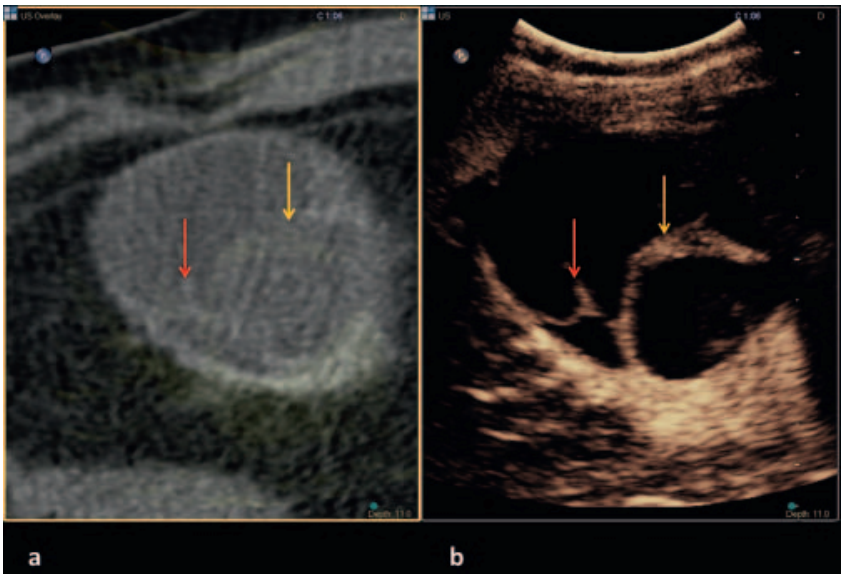

- Fig. 2 Same patient as in $>$ Fig. 1. In the contrast-enhanced venous $\mathrm{CT}$ a, discrete contrast-medium-absorbing septa are visible which also show a discreet contrast-medium-absorption in the contrast-enhanced ultrasound (CEUS) b (red arrows). In addition, however, strong contrast-absorbing septa show up in the CEUS which in the CT show a not so clear differentiability in the corresponding cross-sectional image (yellow arrows). Due to the CEUS this cyst was classified as Bosniak III cyst, the corresponding histology showed a cystic renal cell carcinoma.

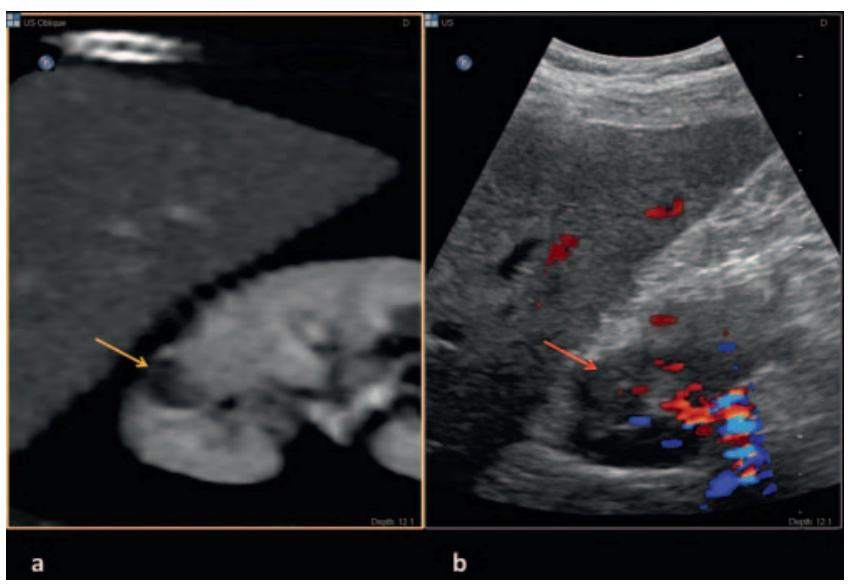

- Fig. 3 The venous phase contrast enhanced CT a shows a cystic lesion at the upper pole of the right kidney without clear CT-morphological evidence of a contrast image. In the B-mode imaging and in the color-coded duplex sonography $\mathbf{b}$ however, a vascularisation of the lesion can already be seen (red arrow).

\section{Results}

Of the 255 patients included in this study with histological clarification of their suspect renal mass, 212 cases were found to be positive (83.1\%); 43 cases reflected negative histopathological findings (16.9\%).

The 212 malignant lesions were distributed as follows: 130 clear cell renal cell carcinomas, 59 papillary renal cell carcinomas, 7 chromophobic renal cell carcinomas, 4 combined clear cell and papillary renal cell carcinomas, and 12 other malignant lesions such as metastases. Of the 43 benign lesions a total of 10 angiomyolipomas, 3 oncocytomas, 8 benign renal cysts and

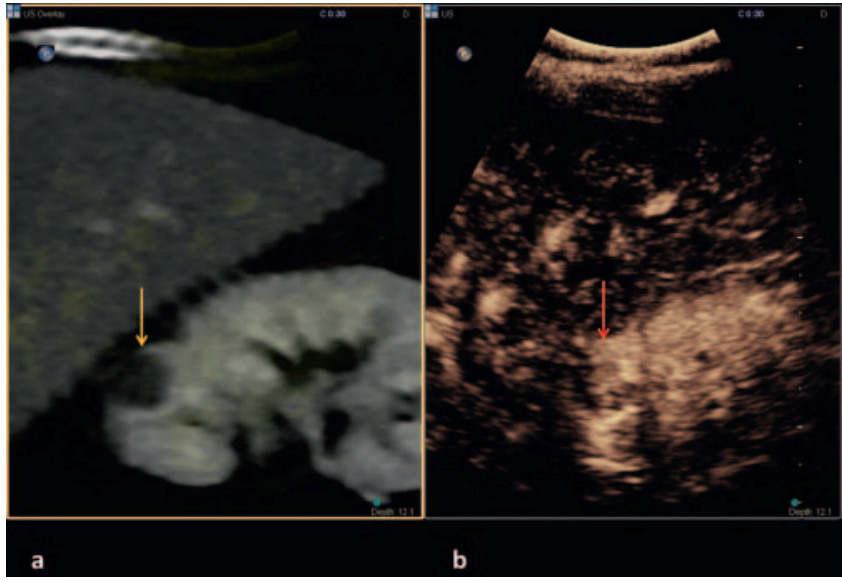

- Fig. 4 Same patient as in $>$ Fig. 3. The cystic lesion (yellow arrow) in the venous phase contrast enhanced CT a shows in the contrastenhanced ultrasound (CEUS) a clear contrast agent absorption in the late arterial phase $\mathbf{b}$, compatible with solid contrast agent absorbing components of the lesion. The lesion was classified as Bosniak 4 by the CEUS. After partial nephrectomy the Histology showed a clear cell renal cell carcinoma.

22 other benign lesions, e. g. renal adenomas, were diagnosed. Using CEUS, 10 lesions were falsely identified as malignant or benign, while 8 lesions were false positive and 2 lesions were false negative. The 8 false-positive lesions included 5 oncocytomas or angiomyolipomas and 3 Bosniak type III cystic lesions.

With the CT scan, 12 lesions were falsely identified as malignant or benign, while 10 lesions were false positive and 2 lesions were false negative. Eight of 10 incorrectly diagnosed lesions using CT were oncocytomas or angiomyolipomas. With the MRI, 3 lesions were falsely identified as malignant or benign, while 2 lesions were false positive and 1 lesion was false negative. All incorrectly diagnosed lesions in the MRI were oncocytomas or angiomyolipomas.

Among the renal lesions histopathologically classified as malignant, the size was on average $2.3 \mathrm{~cm}$ with a range of 0.7 to $7.8 \mathrm{~cm}$. The average size of the benign lesions was $1.8 \mathrm{~cm}$ with a range of 0.8 to $6.3 \mathrm{~cm}$. The eight false-positive findings and the three Bosniak category III renal lesions lay within a range of 0.08 to $3.2 \mathrm{~cm}$ and 1.4 to $3.6 \mathrm{~cm}$ respectively. Both false-negative findings concerned lesions 0.8 and $0.9 \mathrm{~cm}$ in size.

The CEUS showed a sensitivity of $99.1 \%$ (95\% Cl: $96.7 \%$, 99.9\%), a specificity of $80.5 \%$ (95\% Cl: $65.1 \%, 91.2 \%)$, a positive predictive value (PPV) of $96.4 \%(95 \% \mathrm{Cl}: 93.0 \%, 98.4 \%)$ and a negative predictive value (NPV) of $94.3 \%$ (95\% Cl: $80.8 \%, 99.3 \%$ ).

The CT showed a sensitivity of $97.1 \%$ (95\% Cl: $89.9 \%, 99.6 \%$ ), a specificity of $47.4 \%$ ( $95 \% \mathrm{Cl}: 24.4 \%, 71.1 \%$ ), a positive predictive value (PPV) of $87.0 \%$ (95\% Cl: $77.4 \%, 93.6 \%)$ and a negative predictive value (NPV) of $81.8 \%$ (95\% Cl: $48.2 \%, 97.7 \%)$.

The MRI showed a sensitivity of $96.4 \%$ (95\% Cl: $81.7 \%$, 99.9\%), a specificity of $75.0 \%$ (95\% Cl: $34.9 \%, 96.8 \%$ ), a positive predictive value (PPV) of $93.1 \%$ (95\% Cl: 77.2\%, 99.2\%) and a negative predictive value (NPV) of $85.7 \%$ (95\% Cl: $42.1 \%$, 99.6\%). 


\section{Conclusions}

Incidentally-discovered kidney lesions represent a multimodal challenge in diagnosis with far-reaching consequences for further patient management. Although most malignant lesions can be diagnosed preoperatively with sufficient certainty, due to uncertain imaging results, some benign lesions are further clarified interventionally or surgically removed. Ambiguous kidney lesions can be clarified with a high PPV and NPV using CEUS. The additional use of CEUS for ambiguous renal parenchymal lesions may facilitate diagnosis and reduce the number of biopsies and surgical removal as well as validate malignancy in lesions that might otherwise have only been finally diagnosed in further follow-up examinations.

\section{Discussion}

This retrospective study demonstrated that CEUS was shown to have a high PPV (96.4\%), good specificity (80.5\%) and excellent sensitivity (99.1\%) for determination of the tumor characteristics of an ambiguous renal lesion. Overall, this is comparable with other imaging procedures such as CT or MRT, and these results are consonant with several earlier studies on this topic [21, 2628]. Based on these study results, ambiguous renal lesions can already be classified with extremely high accuracy with regard to PPV and NPV on the basis of the morphological character in CEUS imaging, which with respect to NPV can spare the patient an FNA/ biopsy or surgery and their attendant risks.

In the present study, primarily oncocytomas and angiomyolipomas were misinterpreted, which may be consistent with other studies that show similar difficulties in differentiating these entities due to similar imaging characteristics [29-36]. The literature describes how the additive performance of shear wave elastography with ARFI (acoustic radiation force impulse) can be used to obtain better differentiation between clear cell renal cell carcinomas and oncocytomas. This is a dynamic examination which analyses the displacement of the tissue to be examined in order to obtain additional information about the mass in question [37].

CEUS is a good alternative for the precise characterization of ambiguous kidney lesions in patients with e. g. acute or chronic renal failure, hyperthyroidism, or metal implants that are not suitable for MRI or in whom an allergic reaction to iodine or gadolinium is known [38]. In addition, CEUS offers the advantage that non-ionizing radiation unlike in CT and, compared to MRI, there is much wider availability and is also much more cost-effective. With respect to the broad availability in all age groups, radiation hygiene and the side effect profile of the contrast agents used, CEUS with its high specificity offers a good alternative in the further clarification of ambiguous kidney lesions, in follow-up imaging and regarding cost-effectiveness while avoiding surgical resections.

Furthermore, CEUS represents a dynamic examination technique with the possibility of repeating the administration of contrast agents due to the characteristic features of the contrast agents used, which do not affect kidney, thyroid and liver function. The high PPV and NPV of CEUS could reduce the number of necessary CT examinations with associated radiation exposure, the use of contrast agents with renal toxicity and, with regard to MRI, the economic burden on the healthcare system.

However, this study was limited by several factors. Primarily this was a retrospective single-center study in which the CEUS investigations were performed by a single experienced radiologist (EFSUMB Level 3). Various imaging devices were used; furthermore the quantities of contrast agents used varied. The $C T$ and MRI protocols and imaging series also varied in patients with respect to the protocols and scanners available at the time of the examination, which had a significant influence, especially on the specificity of CT and MRI diagnostics. For example, a differentiation between subtypes of renal cell carcinoma and oncocytomas can be achieved by specifically-adapted MRI protocols with a specificity of $97 \%$ as stated in the literature [39].

In this study, only a relatively small percentage (16.9\%) of all image morphologically unclear lesions were classified as benign in the histopathological work-up, which is significantly lower compared to the expected $45 \%$ from national statistics [40], this, however is possibly due to the pre-selected study cohort of a university hospital [36]. If in the initial CT or MRI report the results did not allow a clear statement about the malignancy, the findings of the CT or MRI examinations were equated to the corresponding CEUS examination, which was a necessity in the statistical evaluation, but possibly distorted the statement to a low percentage. Furthermore, CT and MRI imaging could not be obtained for all patients, which meant that the total number of these examinations was significantly lower compared to CEUS.

\section{CLINICAL RELEVANCE OF THE STUDY}

- CEUS is a widely available diagnostic tool that can be used in addition to established imaging procedures such as CT and MRI to distinguish between malignant and benign renal lesions.

- It demonstrates comparable sensitivity, specificity, PPV and NPV to CT and MRI and can help to reduce the number of biopsies and/or surgical interventions.

- In daily clinical routine, CEUS can particularly benefit with contraindications for other imaging procedures.

\section{Conflict of Interest}

The authors declare that they have no conflict of interest.

\section{References}

[1] Chow WH, Devesa SS, Warren JL et al. Rising incidence of renal cell cancer in the United States. JAMA 1999; 281: 1628-1631

[2] Landis SH, Murray T, Bolden S et al. Cancer statistics, 1999. CA Cancer J Clin 1999; 49: 8-31

[3] Hock LM, Lynch J, Balaji KC. Increasing incidence of all stages of kidney cancer in the last 2 decades in the United States: an analysis of surveillance, epidemiology and end results program data. J Urol 2002; 167: 57-60 
[4] Motzer RJ, Hutson TE, Tomczak P et al. Sunitinib versus interferon alfa in metastatic renal-cell carcinoma. N Engl J Med 2007; 356: 115-124

[5] Wallen EM, Pruthi RS, Joyce GF et al. Kidney cancer. J Urol 2007; 177: 2006-2018; discussion 18-19

[6] Decastro G], McKiernan JM. Epidemiology, clinical staging, and presentation of renal cell carcinoma. Urol Clin North Am 2008; 35: 581-592

[7] Woldrich JM, Mallin K, Ritchey J et al. Sex differences in renal cell cancer presentation and survival: an analysis of the National Cancer Database, 1993-2004. J Urol 2008; 179: 1709-1713; discussion 13

[8] Bosniak MA. The small (less than or equal to $3.0 \mathrm{~cm}$ ) renal parenchymal tumor: detection, diagnosis, and controversies. Radiology 1991; 179: 307-317

[9] Rubenthaler J, Bogner F, Reiser M et al. Contrast-Enhanced Ultrasound (CEUS) of the Kidneys by Using the Bosniak Classification. Ultraschall in Med 2016; 37: 234-251

[10] Ljungberg B, Cowan NC, Hanbury DC et al. EAU guidelines on renal cell carcinoma: the 2010 update. Eur Urol 2010; 58: 398-406

[11] Greis C. Technology overview: SonoVue (Bracco, Milan). Eur Radiol 2004; 14 (Suppl. 8): P11-P15

[12] Greis C. Ultrasound contrast agents as markers of vascularity and microcirculation. Clin Hemorheol Microcirc 2009; 43: 1-9

[13] Greis C. Summary of technical principles of contrast sonography and future perspectives. Radiologe 2011; 51: 456-461

[14] Piscaglia F, Bolondi L. Italian Society for Ultrasound in M et al. The safety of Sonovue in abdominal applications: retrospective analysis of 23188 investigations. Ultrasound Med Biol 2006; 32: 1369-1375

[15] ter Haar G. Safety and bio-effects of ultrasound contrast agents. Med Biol Eng Comput 2009; 47: 893-900

[16] Clevert DA, Sterzik A, Braunagel $M$ et al. Modern imaging of kidney tumors. Urologe A 2013; 52: 515-526

[17] Reimann R, Rubenthaler J, Hristova P et al. Characterization of histological subtypes of clear cell renal cell carcinoma using contrast-enhanced ultrasound (CEUS). Clin Hemorheol Microcirc 2015; 63: 77-87

[18] Rubenthaler J, Reimann R, Hristova P et al. Parametric imaging of clear cell and papillary renal cell carcinoma using contrast-enhanced ultrasound (CEUS). Clin Hemorheol Microcirc 2015; 63: 89-97

[19] Escudier B, Porta C, Schmidinger M et al. Renal cell carcinoma: ESMO Clinical Practice Guidelines for diagnosis, treatment and follow-updagger. Ann Oncol 2019; 30: 706-720

[20] Houtzager S, Wijkstra H, de la Rosette J] et al. Evaluation of renal masses with contrast-enhanced ultrasound. Curr Urol Rep 2013; 14: 116-123

[21] Barr RG, Peterson C, Hindi A. Evaluation of indeterminate renal masses with contrast-enhanced US: a diagnostic performance study. Radiology 2014; 271: 133-142

[22] Rubenthaler ], Negrao de Figueiredo G, Mueller-Peltzer K et al. Evaluation of renal lesions using contrast-enhanced ultrasound (CEUS); a 10year retrospective European single-centre analysis. Eur Radiol 2018; 28: 4542-4549

[23] Rubenthaler J, Paprottka K, Marcon J et al. Comparison of magnetic resonance imaging (MRI) and contrast-enhanced ultrasound (CEUS) in the evaluation of unclear solid renal lesions. Clin Hemorheol Microcirc 2016; 64: 757-763
[24] Mueller-Peltzer K, Negrao de Figueiredo G, Graf T et al. Papillary renal cell carcinoma in contrast-enhanced ultrasound (CEUS) - A diagnostic performance study. Clin Hemorheol Microcirc 2019; 71: 159-164

[25] Kazmierski B, Deurdulian C, Tchelepi H et al. Applications of contrastenhanced ultrasound in the kidney. Abdom Radiol (NY) 2018; 43: 880898

[26] Clevert DA, Minaifar N, Weckbach S et al. Multislice computed tomography versus contrast-enhanced ultrasound in evaluation of complex cystic renal masses using the Bosniak classification system. Clin Hemorheol Microcirc 2008; 39: 171-178

[27] Gerst S, Hann LE, Li D et al. Evaluation of renal masses with contrast-enhanced ultrasound: initial experience. Am J Roentgenol 2011; 197: 897906

[28] Sanz E, Hevia V, Arias F et al. Contrast-enhanced ultrasound (CEUS): an excellent tool in the follow-up of small renal masses treated with cryoablation. Curr Urol Rep 2015; 16: 469

[29] Forman HP, Middleton WD, Melson GL et al. Hyperechoic renal cel carcinomas: increase in detection at US. Radiology 1993; 188: 431-434

[30] Siegel CL, Middleton WD, Teefey SA et al. Angiomyolipoma and renal cell carcinoma: US differentiation. Radiology 1996; 198: 789-793

[31] Sim JS, Seo CS, Kim SH et al. Differentiation of small hyperechoic renal cell carcinoma from angiomyolipoma: computer-aided tissue echo quantification. J Ultrasound Med 1999; 18: 261-264

[32] Tamai H, Takiguchi Y, Oka M et al. Contrast-enhanced ultrasonography in the diagnosis of solid renal tumors. J Ultrasound Med 2005; 24: 16351640

[33] Fan L, Lianfang D, Jinfang $X$ et al. Diagnostic efficacy of contrast-enhanced ultrasonography in solid renal parenchymal lesions with maximum diameters of $5 \mathrm{~cm}$. J Ultrasound Med 2008; 27: 875-885

[34] Haendl T, Strobel D, Legal W et al. Renal cell cancer does not show a typical perfusion pattern in contrast-enhanced ultrasound. Ultraschall in Med 2009; 30: 58-63

[35] Wei SP, Xu CL, Zhang Q et al. Contrast-enhanced ultrasound for differentiating benign from malignant solid small renal masses: comparison with contrast-enhanced CT. Abdom Radiol (NY) 2017; 42: 2135-2145

[36] Klezl P, Kaspar M, Klecka J et al. The role of contrast-enhanced pulse inversion harmonic imaging (CEUS) and contrast-enhanced computed tomography (CECT) in the preoperative diagnosis of renal lesions. Urologe A 2018; 57: 181-190

[37] Thaiss WM, Bedke J, Kruck S et al. Can contrast-enhanced ultrasound and acoustic radiation force impulse imaging characterize CT-indeterminate renal masses? A prospective evaluation with histological confirmation. World J Urol 2019; 37: 1339-1346

[38] Rubenthaler ], Wilson S, Clevert DA. Multislice computed tomography/ contrast-enhanced ultrasound image fusion as a tool for evaluating ^unclear renal cysts. Ultrasonography 2019; 38: 181-187

[39] Cornelis F, Lasserre AS, Tourdias T et al. Combined late gadolinium-enhanced and double-echo chemical-shift MRI help to differentiate renal oncocytomas with high central T2 signal intensity from renal cell carcinomas. Am J Roentgenol 2013; 200: 830-838

[40] Reuter VE, Presti JC Jr. Contemporary approach to the classification of renal epithelial tumors. Semin Oncol 2000; 27: 124-137 